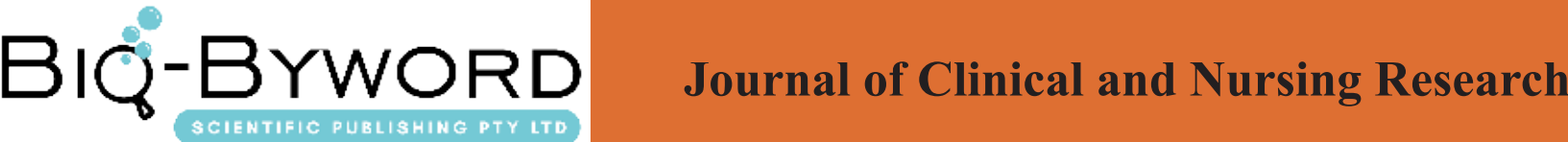

\section{Effect of Rat C6 Glioma Cells in Varying Concentrations of Cultured in Isorhamnetin}

\author{
YANG Jin-hua ${ }^{1}$ SUN Yang-ni* YANG Xiang-li ${ }^{2}$ TIAN Rui-rui ${ }^{3}$
}

Shaanxi Xi'an West Group Hospital, Xi'an, Shaanxi 710077

\section{ARTICLE INFO}

Article history:

Published online: 15th July, 2017

Key words:

Isorhamnetin

glioma cells

apoptosis

$\mathrm{PI} 3 \mathrm{~K} / \mathrm{AKT}$
*Corresponding author

Sun Yang-ni, Shaanxi Xi'an West Group Hospital, Xi'an, Shaanxi 710077. Email: szn080510@163.com

\section{ABSTRACT}

Objective: To investigate the effects of different concentrations of isorhamnetin on C6 rat glioma cells in vitro from January 2015 to June 2015. Methods: The blank control group, blank solvent control group and four concentration groups were used to observe the cell growth status under a microscope. MTT colorimetric assay was used to detect the effect of isorhamnetin on C6 glioma cells in vitro and the cell inhibition rate And survival rate were measured. The apoptotic and apoptotic rates were measured by flow cytometry in the treatment group and the control group. The relationship between the different concentrations of isorhamnetin and C6 glioma cell apoptosis was analyzed the total protein was extracted and the total AKT protein and Ser473 AKT protein content were detected by Western blotting. The rat model of glioma was constructed by SD rats. Five days of isorhamnetin was continuously fed and the plasma was detected by high-performance liquid chromatography, liver, brain tissue isorhamnetin content. Results: Inverted microscopy and image analysis were observed under the observation of the application of isorhamnetin, the tumor cells showed significant changes in apoptosis and necrosis. MTT colorimetric method showed that the higher the concentration of isorhamnetin, the worse the proliferation rate of $\mathrm{C} 6$ glioma cells in vitro, the higher the inhibition rate, the lower the survival rate. Apoptosis was observed by flow cytometry. The apoptosis rate of C6 glioma cells treated with isorhamnetin at the concentration of $40 \mu \mathrm{g} / \mu \mathrm{l}$ was the highest. Add $80 \mu \mathrm{g} / \mu \mathrm{l}$ of isorhamnetin after treatment, the viability of C6 glioma cells was the lowest. 
The expression of AKT protein and AK47 protein in the total AKT and Ser473 loci were significantly higher than those in the control group. The plasma and brain tissues of the rats were detected by high-performance liquid chromatography (HPLC), indicating that there was allogeneic isorhamnetin in the area of plasma, and the distribution of isorhamnetin in plasma and brain tissues were mainly in the brain tissue. Conclusion: Low concentration of isorhamnetin can induce the apoptosis of C6 glioma cells. High concentration of isorhamnetin can induce apoptosis and necrosis of C6 glioma cells in vitro, and inhibit the proliferation of glioma cells And the mechanism of PI3K/ AKT pathway was closely related. In the SD rat brain glioma model, the samples were processed and the plasma and brain tissues were detected by high-performance liquid chromatography (HPLC) Li Su has the target of brain tissue, and provides an experimental argument for the development and utilization of isorhamnetin in anti-tumor.

\section{Introduction}

In recent years, some artifacts such as artemisinin have been well developed for antitumor drugs. Plant-derived drug monomers or compounds have great potential for the discovery of new drugs, such as providing new ideas for designing ideal new drug structures, and can also be used as a lead compound for the creation of new drugs. Isorhamnetin (isorhamnetin, ISO) belongs to the flavonoids, in recent years found that it has in addition to cough expectorant, digestion stagnation, blood stasis, there is better anti-myocardial hypoxia, anti-Arrhythmia, antioxidant free radicals, ischemia, relieve angina, lower serum cholesterol and other cardiovascular effects, the current clinical only for cough expectorant, digestion stagnation, blood stasis. In recent years, studies have found that isorhamnetin on some tumor cells have a significant effect ${ }^{[1-2]}$, also found that isorhamnetin on the inhibition of the tumor has a certain selectivity ${ }^{[3]}$, such as can inhibit in vitro The proliferation and differentiation of A549 and MCF-7 cells have a certain effect on the differentiation of lung cancer and gastric cancer cells ${ }^{[4-5]}$. The proliferation and differentiation of PC3, K562 and AD293 cells have no effect. Lenin et al. ${ }^{\left[{ }^{[6]}\right.}$ have mentioned the relationship between isorhamnetin and mouse glioma cells in the experiment, but I have consulted a large number of literature that have not been reported on the effect of isorhamnetin on glioma cells The detailed test and mechanism of action. This study was to investigate the effect of different concentrations of isorhamnetin on rat $\mathrm{C} 6$ glioma cells in vitro and to provide an experimental basis for in vitro and in vivo studies of isorhamnetin.

\section{Materials and methods}

\subsection{Materials}

Experimental cell lines: C6 glioma cells were purchased from Shanghai Institute of Cell Sciences, Chinese Academy of Sciences.

Experimental rats: 20 SD rats, male and female half, an average of $300 \mathrm{~g}$ each by the Institute of Experimental Animal Center (SPF level) to provide.

Cell test reagents: DMEM medium was purchased from Gibco Corporation, 10\% fetal bovine serum (Sijiqing Biology Co., Ltd.), dimethyl sulfoxide (Chongqing Chemical Reagent Factory), isorhamnetin (Shenzhen Tongtian Biochemical Technology Co., Ltd.) ), 0.25\% trypsin (Hyclone), cell proliferation and cytotoxicity MTT assay kit, Annexin V-FITC / PI double staining cell apoptosis detection kit were purchased from Shanghai Yan Bin company, AKT and Ser473 site AKT protein (Cell Signaling, import), cell culture dish purchased from Hangzhou Shengyou Biotechnology Co., Ltd., Tissue Protein Extraction Kit was purchased from Beijing Baxter Company. 
Cell experiments Main instruments: Flow cytometry (SL), High-speed refrigerated centrifuge (universal 32R, Germany), Inverted microscope (Olympus, Japan), Ultraviolet spectrophotometer (Auriud CE2401, UK), Gel image analysis system instrument (Touching 995gel document system, Tian Cheng Technology Companies), Electrophoresis instrument (FC101, Dalian), Ultra-low temperature refrigerator $\left(-80^{\circ} \mathrm{C}\right)$ (Sanyo, Japan), Incubator (NuAIRE, USA), Ultra pure water production (Milli-Q).

\subsection{Methods}

Preparation of different concentrations of isorhamnetin solution: isorhamnetin (Shenzhen Tong Tian Biochemical Technology Co., Ltd. produced), white powder solid, showing a purity of $98 \%, 10 \mathrm{mg} /$ support. Drugs with $0.1 \% \mathrm{NaOH}$ dissolved into the mother liquor, and then diluted with DMEM complete culture medium, the final concentration of $1 \mu \mathrm{mol} / \mu \mathrm{l}$, filter sterilization after 4 stand.

Drug treatment and cell morphology observation: good growth rat C6 glioma cells were diamond-shaped, three-dimensional strong, less intracellular particles, the edge of the whole, clear structure, strong refraction. A typical rat $\mathrm{C} 6$ glioma cells were seeded in a six-well plate $(2 \mathrm{ml}$ solution per well). After the cell density in the six-well plate was $80 \%$, the cells were divided into three groups: blank control group, DMSO solvent control group, the cells were treated with the control group $(10,20,40,80 \mathrm{mg} / \mathrm{L}$ isorhamnetin). After 48 hours of culture, the growth and morphological changes of the cells were observed under microscope.

MTT assay growth inhibition rate: set blank $0.1 \%$ $\mathrm{NaOH}$ control. The blank control group was supplemented with $0.1 \% \mathrm{NaOH}$ and DMEM complete culture medium, and cultured in $37^{\circ} \mathrm{C}, 5 \% \mathrm{CO}_{2}$ incubator. Take the rat $\mathrm{C} 6$ glioma cells in logarithmic growth phase, and the cells were adjusted to $5 \times 104 / \mathrm{L}$ in 96 -well culture plates with $100 \mu \mathrm{L}$ per well and three wells. The rats in the experimental group were given 10, 20, 40, $80 \mathrm{mg} / \mathrm{L}$ isorhamnetin and 10\% DMEM complete culture medium without cell as the blank control and solvent control. The MTT was completely diluted with the final medium $10 \mu \mathrm{l}$ was added to each well, and the cells were placed in a $5 \% \mathrm{CO}_{2}$ incubator for 4 hours and then aspirated with MTT medium. $150 \mu \mathrm{L}$ of lysate was added to each well at $490 \mathrm{~nm}$ the absorbance of each well was measured. The inhibitory rate of the treated cells was calculated by comparing the absorbance of the untreated cells with $100 \%$ control.
Flow cytometry was used to detect the apoptosis of the cells. After incubation for $72 \mathrm{~h}$, the cells were washed and discarded once a day. After adding $0.25 \%$ trypsin for $1 \mathrm{~min}$. Beat into a suspension after inhalation centrifuge tube, $1200 \mathrm{rpm}$ centrifuge $3 \mathrm{~min}$. Discard the supernatant, add $2 \mathrm{mlPBS}$ rinse $1200 \mathrm{rpm}$ centrifuge $3 \mathrm{~min}$, and then repeat. Discard the supernatant after centrifugation and add $400 \mu \mathrm{l}$ of the binding solution in the Apoptosis Detection Kit. After blowing, add $5 \mu$ of Annexin-v-FITC dye solution to each tube. After incubation at $4{ }^{\circ} \mathrm{C}$ for $15 \mathrm{~min}, 10 \mu \mathrm{l}$ of $10 \mu \mathrm{l}$ of propidium iodide (P1) staining solution ( $20 \mu$ g.m1-1) was incubated at $4{ }^{\circ} \mathrm{C}$ for $5 \mathrm{~min}$. Apoptosis was detected on flow cytometry.

The expression of total AKT protein and Thr473 AKT protein in C6 glioma cells was detected by Western blotting. The cells in the control group and the control group were treated with 24 hours $(2 \times 105$ or more $)$, washed twice with cold PBS, The lysate was lysed on an ice bath for $30 \mathrm{~min}$, centrifuged at $125^{\circ} \mathrm{C}$ for 5 min at $4{ }^{\circ} \mathrm{C}$, and the supernatant was collected and the protein concentration was measured. 10\% SDS-PAGE was used to separate the protein from the nitrocellulose membrane by semi-drying with an electric transducers. Add 1: 2000 dilution of the first anti-reaction for 2.5h, with TBST wash 2 times, then add 1: 1000 diluted AKT and Thr473 site AKT secondary antibody incubation 1 h. Finally ECL is developed and exposed

The rats were divided into three groups: normal control group (group 1), group $2(\mathrm{n}=3)$ and control group (n $=3$ ). The rats were divided into three groups: normal control group, group 2 the rats were sacrificed for 12 $\mathrm{h}$ before administration, and the rats were fed with the control group (50mg / $\mathrm{kg}$ ) for 3 days, $5 \mathrm{~d}, 7 \mathrm{~d}, 10 \mathrm{~d}, 15$ $\mathrm{d}$, dosing 5 times.

The inhibition rate was calculated according to the time of model establishment in rats. On day 3, day 5, day 7 , day 9 and day 12 after rat model, (L), the widest diameter $(\mathrm{L})$, the widest diameter $(\mathrm{W})$, the narrowest diameter $(\mathrm{W})$, the thickness $(\mathrm{H})$, and the mean long diameter $(\mathrm{L})$ and the width of the transplanted tumor were measured. Diameter (W), according to the formula to calculate the volume of transplanted tumor: $\mathrm{V}$ (volume) $=\mathrm{V}$ (volume $)=\mathrm{L} \times \mathrm{W} \times \mathrm{H} \sqrt{2}$. According to the formula: tumor inhibition rate $\%=[$ (negative control group tumor volume - isorhamnetin tumor volume) / negative control group tumor volume] $\times 100 \%$, obtained isorhamnetin macro-tumor inhibition rate.

Histopathological observation of tumor specimens: SD rats were sacrificed at $12 \mathrm{~h}$ after the last (day 12) admin- 


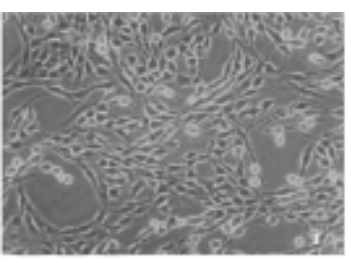

Normal rat

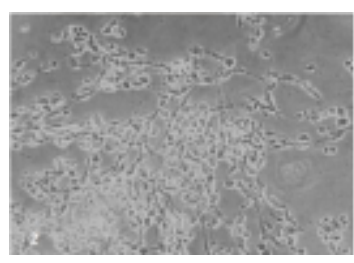

$20 \mu \mathrm{g} / \mathrm{ml}$ Isorhamnetin

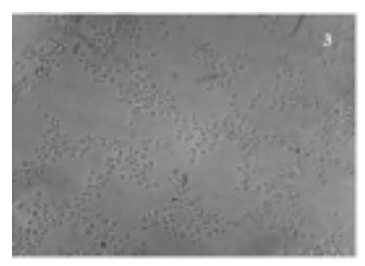

$40 \mu \mathrm{g} / \mathrm{ml}$ Isorhamnetin

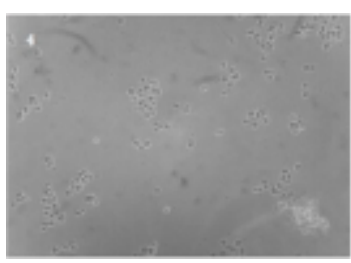

$80 \mu \mathrm{g} / \mathrm{ml}$ Isorhamnetin

Figure 1 Effect of different concentrations of isorhamnetin on the macroscopic morphology of C6 glioma cells $(\times 400)$

istration. Remove the transplanted tumor, half of the rapid into the -80 refrigerator, to be the determination of isorhamnetin content. The other half of the tumor was fixed in $10 \%$ formalin fixative and dehydrated gradually. The tissue was perfused with 4\% PBS paraformaldehyde and fixedly dehydrated. After paraffin embedding, the specimens were cut into $4 \mathrm{~mm}$ coronal slices. HE Dyeing and microscopic observation of brain tumor tissue HE staining.

Determination of Isorhamnetin in Brain Tissue: The transplanted tumor of blank control group and isorhamnetin group was used to determine the distribution of isorhamnetin in the biological samples by isorhamnetin high performance liquid chromatography. The distribution of isorhamnetin in plasma and brain tissue of murine model.

\subsection{Statistical processing}

Using spss13.0 statistical software for analysis, measurement data used to indicate that the count data in terms of rate or percentage. Comparison between the two groups using the mean $t$ test or $\mathrm{X} 2$ test, multiple groups using single factor analysis of variance, correla- tion analysis using the relevance of the data analysis, $\mathrm{P}<0.05$ for the difference was statistically significant.

\section{Results}

2.1 Effects of different concentrations of isorhamnetin on cell morphology

Resuscitation, passage of good growth C6 glioma cell growth: cell viability, $6 \mathrm{~h}$ after the wall can be attached, adherent cells can be seen after the triangle or long prism, cell growth is rapid, $48 \mathrm{~h}$ after the cell can be long Full bottom, that is, into the platform period. After $72 \mathrm{~h}$, some tumor cells were piled up, and no inhibitory growth was observed. After 96 hours, the culture medium had a large number of exfoliated cells. Under the optical microscope, the morphology of monolayer cells is triangular, patterned and polygonal. The cells were stained by $\mathrm{HE}$ and showed long prismatic, irregular polygons, etc. (Fig. 1).

\subsection{MTT colorimetric detection of cell viability}

With the increase of isorhamnetin concentration, C6 glioma cell survival rate decreased gradually, the growth inhibition rate IC50 gradually increased. When the con-

Table 1 Effects of different concentrations of isorhamnetin on C6 glioma cell viability and calculated IC50 values

\begin{tabular}{cccc}
\hline Dose $(\mu \mathrm{g} / \mathrm{ml})$ & Mean OD Value & Cell viability $(\%)$ & $\mathrm{IC}_{50}(\mu \mathrm{g} / \mu \mathrm{l})$ \\
\hline 80 & 0.331 & $64.02 \pm 2.4$ & \\
40 & 0.339 & $65.57 \pm 2.7$ & 76.32 \\
20 & 0.413 & $79.80 \pm 3.5$ & \\
10 & 0.454 & $87.81 \pm 4.1$ & \\
0 & 0.517 & 100.00 & \\
\hline
\end{tabular}


tent of isorhamnetin was $10 \mu \mathrm{g} / \mu \mathrm{l}, 20 \mu \mathrm{g} / \mu \mathrm{l}, 40 \mu \mathrm{g} /$ $\mu \mathrm{l}, 60 \mu \mathrm{g} / \mu \mathrm{l}$ and $80 \mu \mathrm{g} / \mu \mathrm{l}$, the cell viability (\%) was $87.81,79.80,65.57,64.02$. The results showed that isorhamnetin inhibited the growth of C6 glioma cells in a certain concentration-dependent manner, the higher the concentration, the more obvious inhibition. The IC50 of C6 glioma cells was approximately $76.32 \mu \mathrm{g} / \mu \mathrm{l}$ for $48 \mathrm{~h}$ of glioma cells in C6 glioma cells, and the inhibitory rate of C6 glioma cells was plotted as the abscissa. 3, * t-test, $\mathrm{P}<0.05)$.

2.3 The apoptotic rate and apoptotic peak were detected by apoptotic assay

As shown in Fig. 2, the apoptosis rate of the apoptotic tumor cell area was $5.83 \%$ in the blank control group and $88.0 \%$ in the normal tumor cell area. The apoptotic rate of tumor cells was $14.0 \%$, and the cell survival rate of normal cells was $80.4 \%$. The apoptotic rate of tumor cells was $16.7 \%$, and the cell survival rate was $76.7 \%$ in the normal cells. The apoptosis rate of $80 \mu \mathrm{g} / \mu \mathrm{l}$ of isorhamnetin was $19.3 \%$, and the cell survival rate was $75.4 \%$ in normal tumor cell area. The results showed that the apoptosis rate of C6 glioma cells increased with

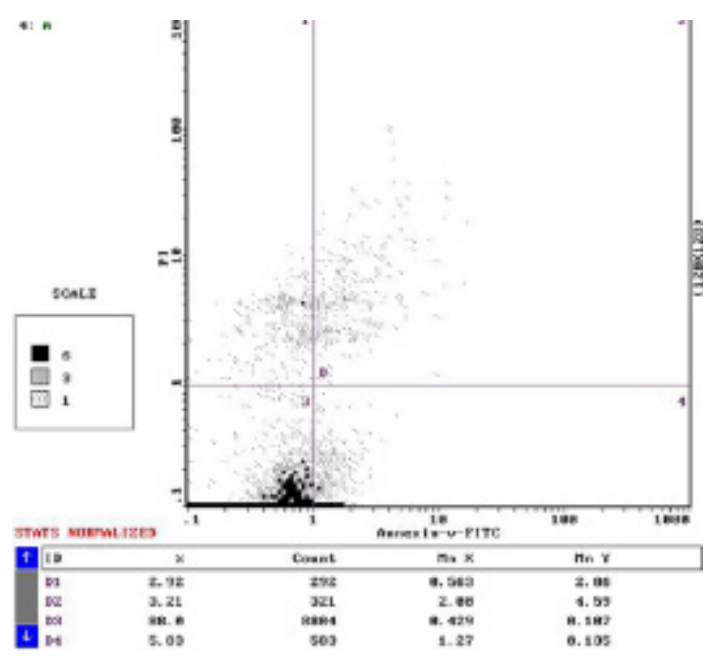

Blank control group

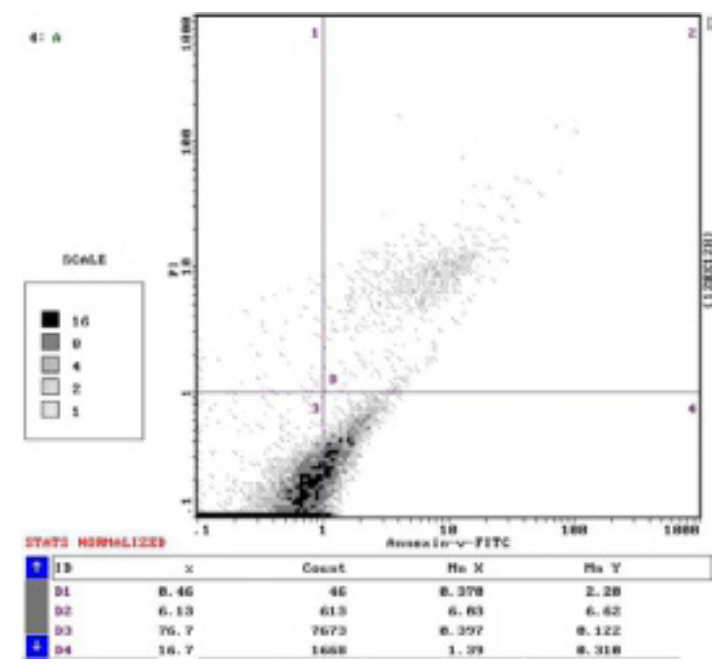

$40 \mu \mathrm{g} / \mathrm{ml}$ isorhamnetin

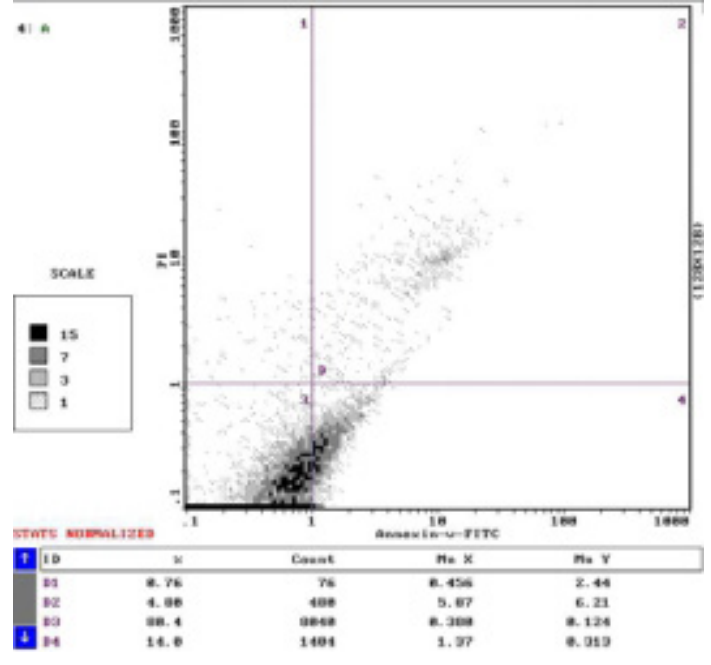

$20 \mu \mathrm{g} / \mu \mathrm{l}$ isorhamnetin

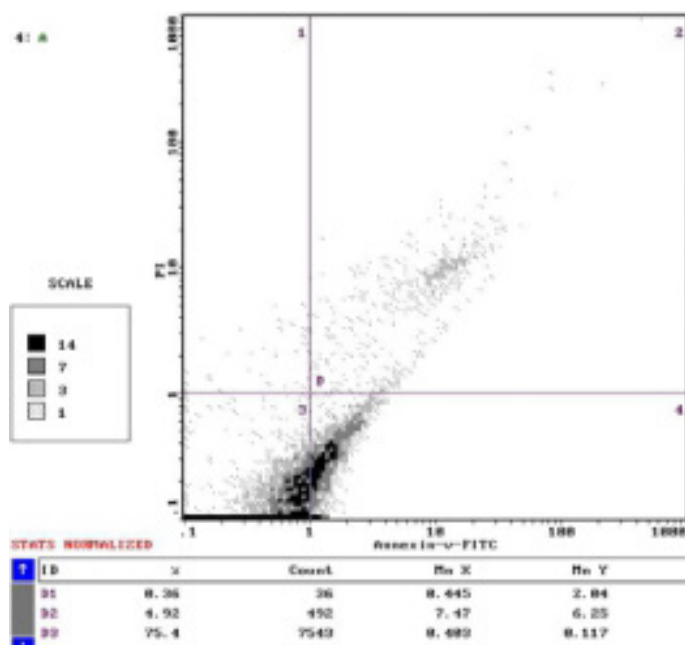

$80 \mu \mathrm{g} / \mathrm{ml}$ isorhamnetin

Figure 2 Effect of different concentrations of isorhamnetin on C6 glioma cell apoptosis 


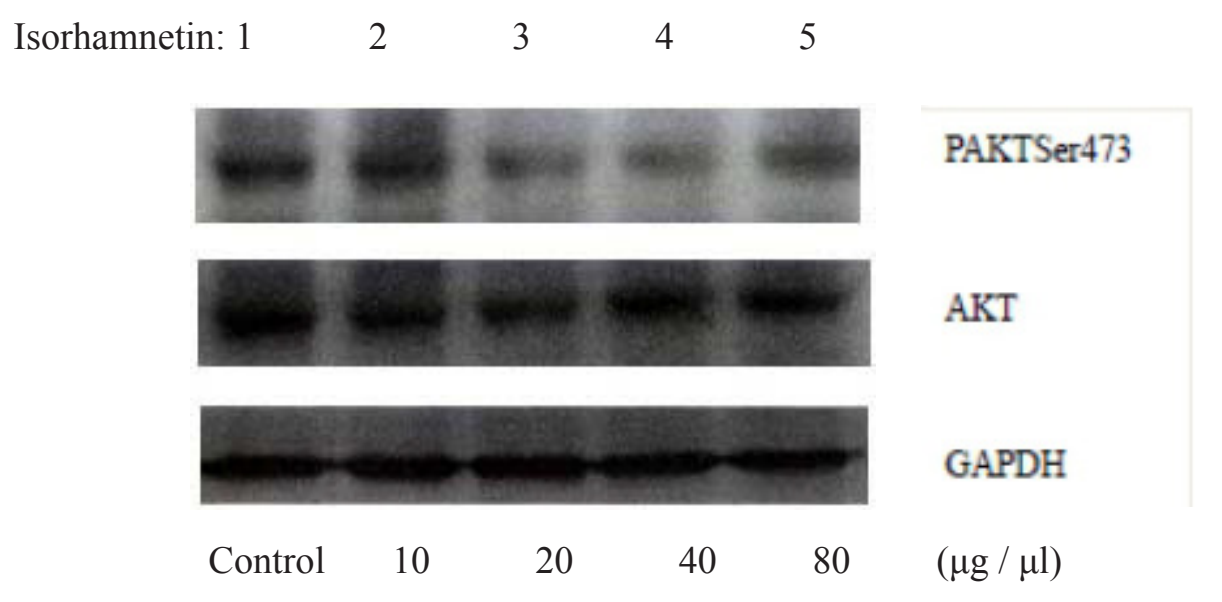

Figure 3 Effect of different concentrations of isorhamnetin on expression of AKT and pAKT

(Ser473) protein in C6 glioma cells

the increase of isorhamnetin concentration.

The area of D1 is normal tumor cell area, D2 region is necrotic tumor cell area, D3 region is necrosis and apoptosis of tumor cell area, D4 area for the apoptosis of tumor cell area,

\subsection{SDS - PAGE Western blot analysis}

Compared with the control group, the pAKT (Ser473) protein band was the deepest and the pAKT (Ser473) was the highest in the four groups. In the group of $10 \mu \mathrm{g}$ / $\mu$ of isorhamnetin PAKT (Ser473) protein was stronger than that of pAKT (Ser473) protein. The protein of pAKT (Ser473) was more than that of $20 \mu \mathrm{g} / \mu \mathrm{l}$ of isorhamnetin. The protein group of pAKT (Ser473) was also lighter than that of the control group. The protein band of pAKT (Ser473) was lighter in the protein group with $80 \mu \mathrm{g} / \mu \mathrm{l}$ of isorhamnetin. The results showed that the pAKT (Ser473) protein was a gradually decreasing trend with the increase of the concentration of isor- hamnetin. The total AKT protein band in the cells was almost no difference.

\subsection{Rat model brain tissue sections}

Figure 4, C6 model rats administered brain tissue HE stained sections of deep red for the growth of good tumor tissue, dark red middle of the hollow tissue atrophy, light red for the normal brain tissue. In the blank control model, the brain tissue sections of the rats showed obvious intercellular connection, good nucleus refractive index, clear cell morphology, polygonal, triangular, climbing. The rate of cell refraction was significantly lower than that of the blank control group, and there was no obvious difference between the cells. There was no significant difference between the cells. The cell shape was round and the cell edge was not obvious.

\subsection{Rat plasma and brain tissue}

As shown in Table 2, isorhamnetin reference substance, the peak time is $13.71 \mathrm{~min}$, the peak area of

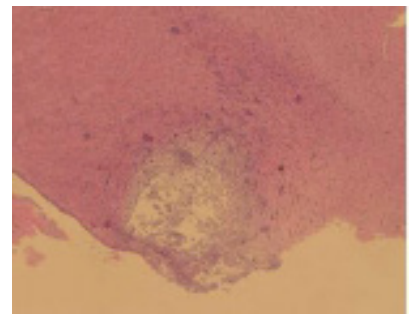

C6 modeling treated rats

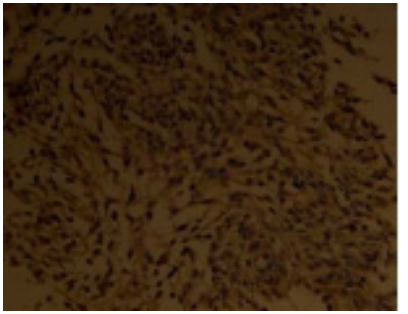

Blank control group

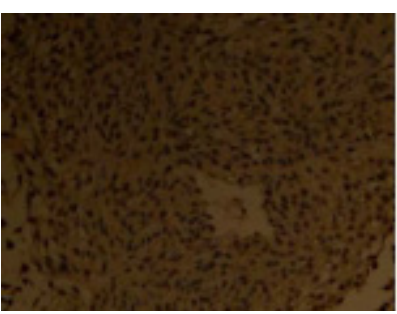

Continuous feeding of isorhamnetin

Figure 4 Observation of tumor tissue under light microscope 
Table 2 High-performance liquid chromatography determination of rat plasma and brain tissue isorhamnetin peak area

\begin{tabular}{cccccc}
\hline & 1 & 2 & 3 & 4 & Average \\
\hline $\begin{array}{c}\text { Concentration of isorhamine } \\
\text { reference substance }\end{array}$ & 384.46 & 400.31 & 396.92 & 371.52 & 388.30 \\
$\begin{array}{c}\text { Brain tissue detection 15min } \\
\text { peak area }\end{array}$ & 13.98 & 7.09 & 9.73 & 11.24 & 10.51 \\
$\begin{array}{c}\text { Plasma detection 15min peak } \\
\text { area }\end{array}$ & 0.63 & 2.14 & 1.15 & 4.05 & 1.99 \\
$\begin{array}{c}\text { Kidney test 15min peak area } \\
\text { Liver detection 15min peak }\end{array}$ & 0.00 & 0.00 & 0.00 & 0.00 & 0.00 \\
$\quad 0.01$ & 0.00 & 0.02 & 0.01 & 0.01 \\
$\begin{array}{c}\text { Prea } \\
\text { Percentage of plasma distri- } \\
\text { bution }\end{array}$ & & & & & $2.7 \%$ \\
$\begin{array}{c}\text { Percentage of brain tissue } \\
\text { distribution }\end{array}$ & & & & $0.51 \%$ \\
\hline
\end{tabular}

384.46; rat plasma samples of isorhamnetin, peak in the near $15.00 \mathrm{~min}$, more accurate that should be different And then a small peak in the plasma may be different from the rhamnol degradation of the flavonoid compounds; rat brain tumor tissue of isorhamnetin, the peak time of $13.91 \mathrm{~min}$, Peak area is 13.98 ; kidney test results, 15 min no peak, may be different rhamnol metabolism for other monomers; E. liver test results, $15 \mathrm{~min}$ no obvious peak.

\section{Discussion}

The development of chemotherapy drugs, killing tumor cells, the tumor cell apoptosis and necrosis, the treatment of the tumor is very important, the apoptosis is controlled by the initiative, the program of death. Apoptosis is irreversible death, such as DNA fragment fragmentation, nuclear membrane rupture, protein degeneration, enzyme inactivation, causing apoptosis of the drug may also affect the normal cells, but the cells do not relapse after apoptosis, but also from cell proliferation on the inhibition, and to solve the drug resistance of non-apoptotic drugs. Therefore, the study of the relationship between apoptosis and the relationship between the tumors, in-depth study of its mechanism, to seek targeted drugs on the treatment of tumors is important. The results showed that when the concentration of isorhamnetin was greater than $40 \mu \mathrm{g} / \mu \mathrm{l}$, the viability of C6 glioma cells was significantly reduced. Induced apoptosis, inverted microscope observed cell shrinkage, nuclear concentration for a group, the cell volume becomes smaller, the formation of membrane encapsulation atrophy of apoptotic bodies (containing organelles and debris), late nuclear fragmentation, Cell rupture, the number of cells decreased significantly and so on. MTT assay was used to detect the survival rate of C6 glioma cells in vitro. The results showed that the survival rate of C6 cells was decreased with the increase of isorhamnetin concentration. After $48 \mathrm{~h}$ treatment with isorhamnetin, the apoptosis of C6 glioma cells was detected by flow cytometry. The results showed that with the increase of isorhamnetin concentration, the cells gradually moved to the fourth phases. The proportion of normal proliferative cells decreased, four-phase apoptotic cells increased, while the two-phase necrotic cells also increased. Simultaneous results were obtained by different experimental methods. With the increase of isorhamnetin concentration, the survival rate of glioma cells cultured in vitro decreased and the proportion of apoptosis increased, and it was dose-related.

The genes that regulate the normal cycle of cells are divided into proto-oncogenes and tumor suppressor genes. The genes that express the phosphoinositide 3-kinase (PI3K) family are proto-oncogene whose gene expression products can activate 3,4,5-tris Phosphatidylinositol, used as a second messenger in the cell, is the protein kinase PKB (Akt) translocates from 
the cytoplasm to the cell membrane activation signal factor. Cell proliferation and apoptosis of P13K / Akt signaling pathway have a direct relationship, the signal pathway can lead to the occurrence of tumor metastasis, metastasis, invasion [7]. PI3K-activated products PIP2 and PIP3 bind to Akt's platelet-leukocyte c-kinase substrate homozygous region, so that both AKTSer473 and Thr308 phosphorylation are activated and alter the original conformation, To achieve the ultimate goal of Akt activation, and finally transported from the cytoplasm of Akt to the cell membrane, and then the activation of the downstream pathway. Akt activation can activate multiple target sites downstream, such as: Bad, caspase- 9 precursor, mammalian target of rapamycin, mTOR, FKHR / AFX / FOX), Glycogen synthase kinase-3 (GSK-3), p21cipl and Raf ${ }^{[7]}$. Activation of Akt signaling to downstream factors, regulation of information transcription, cell cycle, PI3K / AKT signaling pathway in the cell survival and cell metabolism and so has an unusual role. The results of this study showed that the expression of p-AktSer473 in rat C6 glioma cells was different after adding different concentrations of isorhamnetin. The expression of p-AktSer473 was increased with the addition of isorhamnetin And the expression of p-AktSer473 in rat C6 glioma cells was the lowest after adding isorhamnetin at a concentration of $80 \mu \mathrm{g} / \mu \mathrm{l}$, indicating that $\mathrm{p}$-AktSer473 was expressed in rat $\mathrm{C} 6$ glioma The expression of P-Akt Ser473 was inhibited by the addition of isorhamnetin, and the addition of isorhamnetin was involved in the development and progression of $\mathrm{C} 6$ tumor. More directly, the expression of $\mathrm{p}$-Ak tSer473 protein in C6 glioma cells of rats treated with isorhamnetin was decreased and the apoptotic rate was increased.

In recent years, it has been found that it has the effect of removing cough and expectorant and promoting blood circulation and removing blood stasis, and the addition of rhamnol also has the effect of expanding the content of the traditional Chinese herbal medicine, such as artemisinin, Coronary artery, anti-platelet aggregation anti-tumor and many other pharmacological effects ${ }^{[8-9]}$. Many studies have reported that isorhamnetin inhibits a variety of in vitro cultured tumor cells, but the effect on the glioma has not been reported. This study shows that when the concentration of isorhamnetin is greater than $40 \mu \mathrm{g} / \mu \mathrm{l}$, Rat C6 glioma cells inhibit the effect is more obvious, while inducing apoptosis, manifested as cell shrinkage, membrane shrinkage, smaller volume, the formation of apoptotic bodies (including apoptotic cell fragments). MTT assay was used to detect the growth inhibition rate and survival rate of rat C6 glioma cells. The results showed that the proliferation of rat C6 glioma cells decreased after $48 \mathrm{~h}$ of isorhamnetin, and the survival rate significantly decreased, the data show that the concentration of isorhamnetin and rat C6 glioma was a certain quantitative relationship. Flow cytometry showed that the survival rate of rat $\mathrm{C} 6$ glioma cells treated with isorhamnetin was $48.4 \%$ and the apoptotic rate was $19.3 \%$, which was $15 \%$ lower than that of negative control group Isorhamnetin has a great potential for promoting rat C6 glioma cells. Western blotting showed that the protein content of AKTSer473 protein was decreased and the protein expression was decreased with the increase of isorhamnetin concentration, which indicated that isorhamnetin could inhibit the activation of AKT protein and enhance the expression of C6 glioma Cell apoptosis. At the same time rat brain glioma in vivo model experiments showed that after 5 consecutive days of feeding isorhamnetin raw material, the rat plasma and brain tissue can be detected in the isorhamnetin monomer, indicating that isorhamnetin on the brain Glioma is a certain targeting, which is isorhamnetin as a therapeutic drug provides a new basis.

When the concentration of isorhamnetin was $40 \mu \mathrm{g} / \mu \mathrm{L}$ and $80 \mu \mathrm{g} / \mu \mathrm{L}$, both MTT and flow cytometry showed that isorhamnetin inhibited rat C6 glioma cells, and the experimental group had the most obvious apoptosis at these two concentrations. When the isorhamnetin concentration of $80 \mu \mathrm{g} / \mu \mathrm{L}$, but also to see the cell membrane rupture, cell edge is unclear, lost the complete cell structure, nucleus shrink or disappear. The results showed that the rat C6 glioma cells had obvious apoptosis under the action of certain concentrations of isorhamnetin, and when the concentration of isorhamnetin reached a certain critical concentration, the rat $\mathrm{C} 6$ brain Glioma cell necrosis. Of course, this is a common, in many anti-tumor drug-induced tumor cell death process, are a small dose of apoptosis, the concentration reaches a certain limit will cause necrosis, high concentrations generally cause apoptosis and necrosis coexistence ${ }^{[10]}$, Because the late apoptosis, when the macrophage load is large enough, cannot quickly remove phagocytic cell debris, can no longer engulf apoptotic bodies, then the cells will occur secondary to necrosis ${ }^{[11-12]}$. From the treatment point of view, if the compounds or monomeric substances that can induce apoptosis are used for clinical treatment, the observation and analysis of the induction action clearly show that isorhamnetin inhibits the growth and induction of C6 glioma cells Apoptosis, and the higher the concentration of the role of the stronger, for the development of isorhamnetin into a new class of anti-glioma drugs laid the experimental basis ${ }^{[13-15]}$. In addition, we should note that in rat brain glioma model experiments, both plasma and brain tissue detection of isorhamnetin, and the distribution of the brain is still relatively impressive, indicating a problem, the brain of 
isorhamnetin Tissue targeting is worthy of recognition, I believe that in the near future, isorhamine can be used as anti-glioma drugs for further development.

In this study, $\mathrm{C} 6$ rat glioma cells were cultured in vitro and the growth of rat $\mathrm{C} 6$ glioma cells was observed by adding different concentrations of isorhamnetin. MTT assay was used to detect the cell proliferation rate. The cells were detected by flow cytometry Apoptotic condition. The relationship between AKT protein and Ser473 AKT protein expression was detected by further study of the relationship between the expression of AKT protein and the PI3K / AKT signal pathway in rat C6 glioma cells. The Value of $\mathrm{Li} \mathrm{Su}$ in the Treatment of Brain Glioma. The survival rate of C6 glioma cells in rats was gradually decreased with the increase of the concentration of isorhamnetin, and the survival rate of C6 glioma cells in rats was decreased with the increase of the concentration of isorhamnetin. The correlation between the mass concentration and the inhibition rate. Isorhamnetin has an apoptosis-inducing effect on C6 glioma cells. The apoptosis rate of rat $\mathrm{C} 6$ glioma cells in different concentrations of isorhamnetin is different, and the apoptosis rate increases with the concentration of isorhamnetin Rise. Detection of protein expression showed that the contents of total AKT protein and Ser473 AKT protein in the cells increased with the increase of the amount of isorhamnetin, and was inversely related. The mechanism of action of isorhamnetin on rat C6 glioma cells is closely related to the PI3K / AKT signaling mechanism. Isorhamnetin into the mouse after the body, mainly distributed in the brain tissue, brain tissue is the main target of isorhamine action.

\section{References}

[1] Cuiyao yuan, Zhou Yiming, Zhang Yu, Zhang Xiaobiao, Li Qiuping, Zhou Hao, Hu Fan, Zhu Wei, Jiang Yingchuan, Yu Yong. Brain Glioma Apoptosis and Related Gene Surviving Expression [J]. Chinese Journal of Neurosurgery Journal, 2003, 19 (6): 448-451.

[2] Shao Naiyuan, Huang Qiang, Dong Jun, Wang Aidong, Lan Qing. Coronary Gyrus Malignant Transformation and Its Differential Expression Gene Analysis [J]. Chinese Journal of Neurosurgery, 2004, 20 (3): 191-195.

[3] Wang Zhengrong, Wang Ling, Yin Huahu, et al. Effect of Total Flavonoids of Hippophae Rhanmoides on Contractile Mechanics and Calcium Transfer in Stretched Myoeyte. [J]. Space Medicine \& Medical Engineering, 2000, 12 (1): 6 -9.

[4] LIN Wei-hong, XIE Xiao-na, CUI Li, ZHANG Bo- min, WANG Shao. Effects of Glucose on C6 Glioma Cell Morphology [J]. Chinese Journal of Laboratory Diagnosis, 2010, 14 (7): 1056-1058

[5] Zhong Xiaogang, Wu Kainan, He Sheng, et al. Effect of Quercetin on the Proliferation and Apoptosis in Transplantation Tumor in Nude Mice [J]. Journal of Sichuan univ (Med Sci Edi), 2003, 34 (3): 439-442.

[6] Alcocer F, Whiteley D, Salazer Gonzaiez JF, et al. Quercetin Inhibits Vascular Smooth Muscle Cell Proliferation and Migration. Surgery, 2002, 131 (2): 198-204.

[7] Jiang BH, Liu LZ. PI3K / PTEN Signaling in Angiogenesis and Tumorigen Is [J]. Adv Cancer Res, 2009, 102: 19- 65 .

[8] Ward S G, Finan P. Isoform-Specific Phosphoinositide 3-Kinase Inhibitors as Therapeutic Agents [J]. Curr Opin Pharmaeol, 2003, 3 (4): 426-434.

[9] Fan Pansheng, Gu Zhenlun, Sheng Rui, et al. Inhibitory Effect Of Quercetin On Proliferation Of Human Nfierovaseular Endothelial Cells In Vitro. Acta Pharmacol Sin, 2003, 24 (12): 1 231-1234.

[10] Zhou Liming, Zhu Ling, Wang Zhengrong. Anti-tumor effect of isorhamnetin in vitro [J]. Chinese Journal of Physiological Sciences, 2004, 26 (4): 191-192.

[11] Zhu Ling, Wang Zhengrong, Zhou Liming, et al. The Effect of Isorhamnetin on Lung Cancer and Its Anti-Tumor Mechanism [J]. Journal of Space Medicine and Medicine, 2005, 18 (5): 381-383.

[12] Wu Qiuge, Jiang Junguang, et al. PI3K / Akt Inhibitor on Lung Adenocarcinoma A549 Cell Proliferation Activity [J]. Chinese Journal of Gerontology, 2011, 3, (1): 45-52.

[13] Boreddy SR, PramankKC, Srivastava S K. Pancreatic Tumor Suppression by Ben Zylisothj0cyanate is associated with Inhibition of PI3K / AKT / FOXO Path Way [J]. Clin CancerRes, 2011, 17 (7): 1784-1795.

[14]He X, Wang Y, Zhu J, et al. Resveratrol Enhances the Anti-Tumor Activity of the mTOR Inhibitor Rapamycin In Multiple Breast Cancer Cell Lines Mainly By Suppressing Rapamycin-Induced AKT Signaling [J]. Cancer Lett, 2011, 301(2) : 168- 176.

[15]Junttila, Robert W. Ligand-Independent HER2/ HER3/PI3K Complex Is Disrupted By Trastuzumab And Is Effectively Inhibited By The PI3K Inhibit Or GDC-0941[J].Cancer Cell,2009, 15(5): 429-440. 